\title{
RESEARCH NOTES
}

\section{A NOTE ON BEST APPROXIMATION AND INVERTIBILITY OF OPERATORS ON UNIFORMLY CONVEX BANACH SPACES}

\author{
by \\ JAMES R. HOLUB

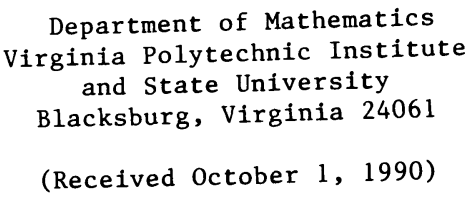

\section{ABSTRACT}

It is shown that if $X$ is a uniformly convex Banach space and $S$ a bounded linear operator on $X$ for which $\|I-S\|=1$, then $S$ is invertible if and only if $\left\|I-\frac{1}{2} S\right\|<1$. From this it follows that if $S$ is invertible on $X$ then either (i) $\operatorname{dist}(I,[S])<1$, or (ii) 0 is the unique best approximation to $I$ from $[S]$, a natural (partial) converse to the well-known sufficient condition for invertibility that $\operatorname{dist}(I,[S])<1$.

Key Words and Phrases: uniformly convex space, invertible operator, unique best approximation.

1980 A.M.S. Subject Classification Codes: 47A05, 47A30, 41 A52.

§1. Introduction. It is well-known [3, p. 584] that if $S$ is a bounded linear operator on a Banach space $X$ for which $\|I-S\|<1$ then $S$ is invertible. Equivalently, if [S] denotes the subspace of $\mathcal{L}(X)$ spanned by $S$, then $S$ is invertible if $\operatorname{dist}(I,[S])<1$. Simple examples show that in the "extreme" case when $\|I-S\|=1$ the operator $S$ may, or may not, be invertible.

In this paper we characterize the invertible operators $S$ on $X$ for which $\|I-S\|=1$ in the case where $X$ is a uniformly convex space (Theorem 1). As a consequence of this result we derive a necessary condition for invertibility of an operator on a uniformly convex space in terms of best approximation to the identity operator in $\mathcal{L}(X)$ which is a natural complement to the sufficient condition cited above (Theorem 2).

The terminology and notation used here is standard (e.g. [3]). For simplicity the word "operator" will be used to mean "bounded linear operator", the word "space" to mean "Banach space", and the symbol $\mathcal{L}(X)$ to denote the space of all operators on $X$. Finally, we recall that a space $X$ is called uniformly convex [2] if for each $0<\epsilon \leq 2$ there exists $0<\delta<1$ so that if $\|x\| \leq 1$, $\|y\| \leq 1$, and $\|x-y\| \geq \epsilon$ in $X$, then $\|x+y\|<2(1-\delta)$; e.g., it is well-known that every $L^{\rho}(\mu)$-space with $1<\rho<+\infty$ is uniformly convex [2]. 
§2. Our results are based on the following recent result of Abramovich, Aliprantis, and Burkinshaw concerning Daugavet's equation in uniformly convex spaces:

THeorem (A-A-B) [1]. : If $X$ is a uniformly convex space, an operator $T$ on $X$ satisfies the equation $\|I+T\|=1+\|T\|$ if and only if $\|T\|$ is in the approximate point spectrum of $T$ (i.e. there is a sequence $\left\{x_{n}\right\}$ in $X$ with $\left\|x_{n}\right\|=1$ for all $n$ for which $\left\|T x_{n}-\right\| T\left\|x_{n}\right\| \rightarrow 0$ ).

From this we have:

Proposition 1. Let $X$ be a uniformly convex space and $T$ an operator on $X$ for which $\|T\|=1$. Then $\|I+T\|<2$ if and only if $I-T$ is invertible on $X$.

Proof: If $I-T$ is invertible then $1=\|T\|$ is not in the approximate point spectrum of $T$, so by Theorem (A-A-B) above $\|I+T\|<2$.

On the other hand, if $\|T\|=1$ and $\|I+T\|<2$ then by Theorem $(\mathrm{A}-\mathrm{A}-\mathrm{B})$ the number 1 is not in the approximate point spectrum of $T$ so the operator $I-T$ must be bounded below on the unit sphere $\{x \mid\|x\|=1\}$ in $X$, and hence $I-T$ is an isomorphism from $X$ onto the closed subspace $\operatorname{ran}(I-T)$ of $X$. If this range of $I-T$ were a proper subspace of $X$ then there would exist a functional $f \in X^{*}$ for which $\|f\|=1$ and $\left(I-T^{*}\right)(f)=0$; but then $T^{*} f=f$, so $\|I+T\|=\left\|I+T^{*}\right\| \geq\left\|\left(I+T^{*}\right)(f)\right\|=2$, a contradiction. Therefore it must be that $\operatorname{ran}(I-T)=X$, and $I-T$ is invertible.

Now, as we remarked earlier, it is well-known that if $S$ is an operator on a space $X$ for which $\|I-S\|<1$ then $S$ is invertible, but if $\|I-S\|=1$ no conclusion is possible. However we now show that in contrast to the general case, if $X$ is uniformly convex we can characterize exactly which such operators are invertible.

TheоReм 1. Let $X$ be a uniformly convex space and $S$ an operator on $X$ for which $\|I-S\|=1$.

Then the following are equivalent:

(i) $S$ is invertible.

(ii) $\left\|I-\frac{1}{2} S\right\|<1$.

(iii) $\|I-t S\|<1$ for all $0<t<1$.

Proof: (i) $\Rightarrow$ (ii). Suppose $S$ is invertible, but $\left\|I-\frac{1}{2} S\right\| \geq 1$. Since $\|I-S\|=1$ it follows that $\left\|I-\frac{1}{2} S\right\|=\frac{1}{2}\|I+(I-S)\| \leq 1$ as well, so $\left\|I-\frac{1}{2} S\right\|=1$ and hence $\|I+(I-S)\|=\|2 I-S\|=2$. But then by Proposition 1 (with $T=I-S$ ) we have that $S=I-(I-S)$ is not invertible, a contradiction. Therefore, if $S$ is invertible it must be that $\left\|I-\frac{1}{2} S\right\|<1$.

(ii) $\Rightarrow$ (iii). Suppose $\left\|I-\frac{1}{2} S\right\|<1$ but $\left\|I-t_{0} S\right\| \geq 1$ for some $0<t_{0}<1$. Again, this implies $\left\|I-t_{0} S\right\|=1$, and hence that $\left\|\left(1-t_{0}\right) I+t_{0}(I-S)\right\|=\|I\|=\|I-S\|=1$. By the Hahn-Banach 
Theorem it follows easily that $\|(1-t) I+t(I-S)\|=1$ for all $0<t<1$ as well, a contradiction to (ii) when $t=\frac{1}{2}$, so (ii) $\Rightarrow$ (iii).

(iii) $\Rightarrow$ (i). If $\|I-t S\|<1$ for all $0<t<1$, then for any such $t$ the operator $t S$ must be invertible by the condition cited above, implying $S$ itself is invertible.

In terms of the geometry of the space $\mathcal{L}(X)$ Theorem 1 has the equivalent formulation:

Corollary 1. If $X$ is uniformly convex, $S \in \mathcal{L}(X)$, and $\|I-S\|=1$, then $S$ is invertible if and only if the open segment $(I, I-S)$ in the unit ball $B$ of $\mathcal{L}(X)$ contains no boundary point of $B$.

Recall, too, that if $X$ is any Banach space and $T \in \mathcal{L}(X)$ satisfies $\|I-T\|<1$, then not only is $T$ invertible, but $T^{-1}$ has the representation

$$
T^{-1}=I+\sum_{n=1}^{\infty}(I-T)^{n},
$$

where this series converges absolutely in $\mathcal{L}(X)[3, \mathrm{p} .584]$. Using this result and Theorem 1 we get the same sort of representation for the inverse of an invertible operator $S$ on a uniformly convex space even when $\|I-S\|=1$.

Corollary 2. Let $X$ be a uniformly convex space and $S$ an invertible operator on $X$ for which $\|I-S\|=1$. Then

$$
S^{-1}=2 I+2 \sum_{n=1}^{\infty}\left(I-\frac{1}{2} S\right)^{n},
$$

where this series converges absolutely in $L(X)$.

Proof: Since $S$ is invertible, by Theorem $1\left\|I-\frac{1}{2} S\right\|<1$. It follows (as above) that $\frac{1}{2} S$ is invertible and $\left(\frac{1}{2} S\right)^{-1}=I+\sum_{n=1}^{\infty}\left(I-\frac{1}{2} S\right)^{n}$, from which the result follows.

Remark: While the assumption of uniform convexity in Theorem 1 is sufficient to imply the conclusions of that theorem, it is possible to weaken this requirement somewhat and still obtain the same results. For example, one can show that if $\boldsymbol{X}$ is only assumed to have a Kadec-Klee norm [4] and $X^{*}$ is strictly convex then Theorem 1 still holds. On the other hand, the fact that some fairly strong geometric conditions must be imposed on $X$ in order to obtain the conclusion of Theorem 1 can be easily seen by examples such as the following:

Example: Let $S: l^{1} \rightarrow l^{1}$ be defined by $S\left(e_{1}\right)=\frac{1}{2} e_{1}+\frac{1}{2} e_{2}$ and $S\left(e_{n}\right)=e_{n}$ for $n \geq 2$, where $\left\{e_{n}\right\}_{n=1}^{\infty}$ denotes the standard basis for $l^{1}$. Clearly $S$ is invertible, $\|I-S\|=\sup \left\|(I-S) e_{n}\right\|=1$, and yet $\left\|I-\frac{1}{2} S\right\|=\sup \left\|\left(I-\frac{1}{2} S\right) e_{n}\right\|=\left\|e_{1}-\frac{1}{2} S e_{1}\right\|=1$ also, so Theorem 1 fails to hold for operators on $l^{1}$.

Now let us return to a consideration of the criterion $\|I-S\|<1$ for invertibility of an operator $S$ on an arbitrary Banach space $X$. Since $S$ is invertible if and only if $\lambda S$ is inveryible for some 
$\lambda \neq 0$, this condition admits the following interpretation in terms of approximation in $\mathcal{L}(\mathrm{Y})$ :

If $[S]$ denotes the subspace of $\mathcal{L}(X)$ spanned by $S$, and if dist $(I,[S])<1$, then $S$ is invertible.

In general, of course, the converse of this result need not hold; however, if $\boldsymbol{X}$ is uniformly convex we can apply Theorem 1 to obtain an interesting partial converse which reveals further the relationship between invertibility of an operator $S$ and best approximation to $I$ from the subspace $[S]$ of $\mathcal{L}(X)$.

Theorem 2. Let $X$ be a uniformly convex space and $S \in \mathcal{L}(X)$. If $S$ is invertible on $X$ then either

(i) $\operatorname{dist}(I,[S])<1$, or

(ii) 0 is the unique best approxiamtion to $I$ from $[S]$.

Proof: Suppose $S$ is invertible on $X$ and $\operatorname{dist}(I,[S]) \geq 1$. Since $\operatorname{dist}(I,[S]) \leq 1$ it must then be that $\operatorname{dist}(I,[S])=1=\|I-0\|$, so 0 is a best approximation to $I$ from $[S]$.

If 0 is not the unique best approximation there is some $\lambda \neq 0$ for which $\|I-\lambda S\|=1$ as well. Since $S$ is assumed to be invertible, $\lambda, S$ is invertible and by Theorem 1 it follows that $\left\|I-\frac{1}{2}(\lambda S)\right\|<1$. But this is a contradiction to the fact that $\operatorname{dist}(I,[S])=1$, so 0 must, in fact, be the unique best approximation, and the result follows.

Remark: Again, the operator $S$ of the example above shows that, in general, Theorem 2 need not hold for an arbitrary space $X$. Exact conditions on $X$ for the validity of Theorem 2 are not known.

\section{REFERENCES}

[1] Y. Abramovich, C. Aliprantis, and O. Burkinshaw, The Daugavet equation in uniformly convex Banach spaces, (to appear).

[2] J. Clarkson, Uniformly convex spaces, Trans. Amer. Math. Soc. 40(1936), p.396-414.

[3] N. Dunford and J. Schwartz, Linear Operators I, Interscience Publishers, New York, NY, 1963.

[4] D. van Dulst and I. Singer, On Kadec-Klee norms on Banach spaces, Studia Math. 54(1975), p.205-211. 


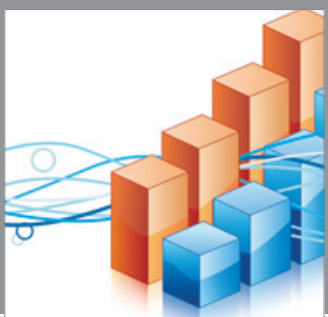

Advances in

Operations Research

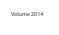

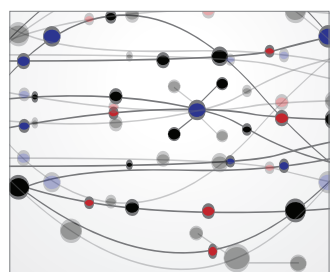

\section{The Scientific} World Journal
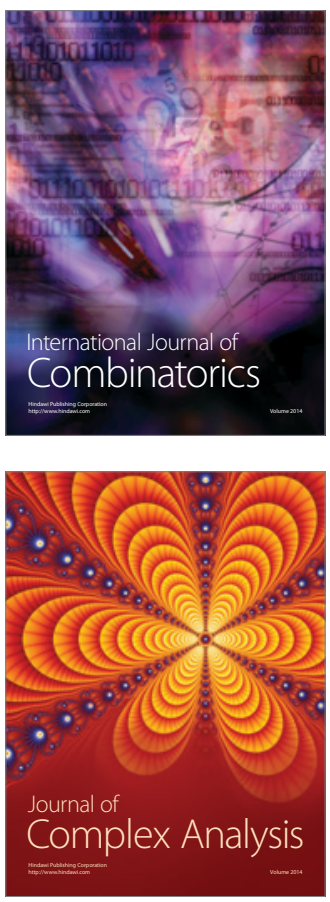

International Journal of

Mathematics and

Mathematical

Sciences
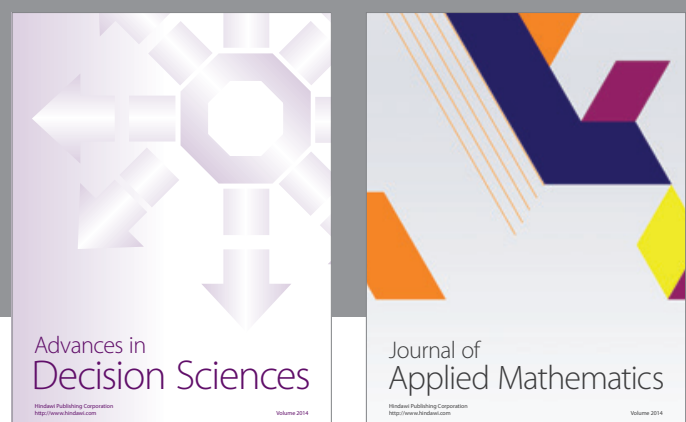

Journal of

Applied Mathematics
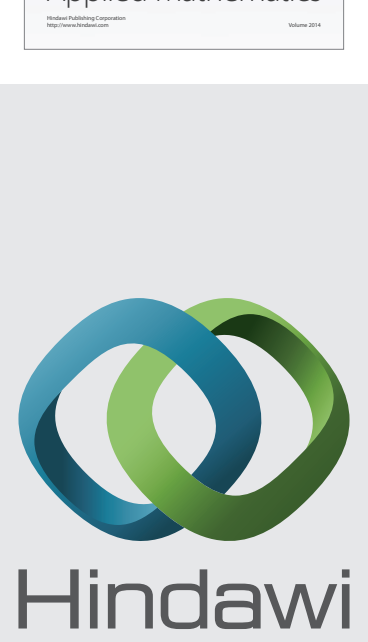

Submit your manuscripts at http://www.hindawi.com
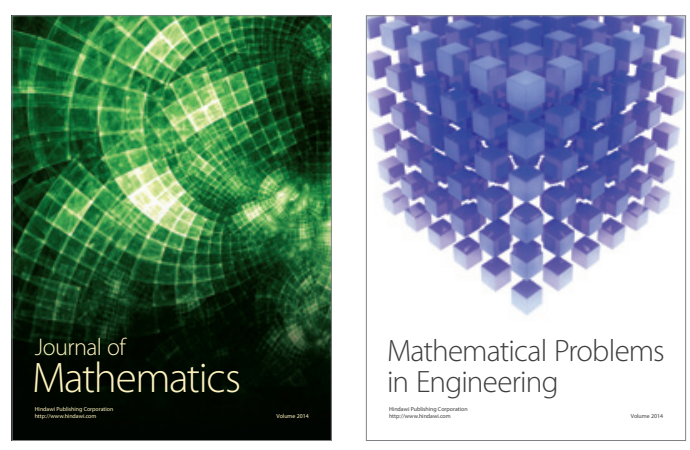

Mathematical Problems in Engineering
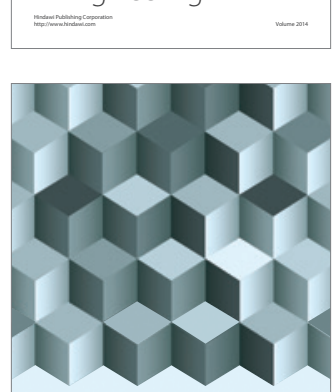

Journal of

Function Spaces
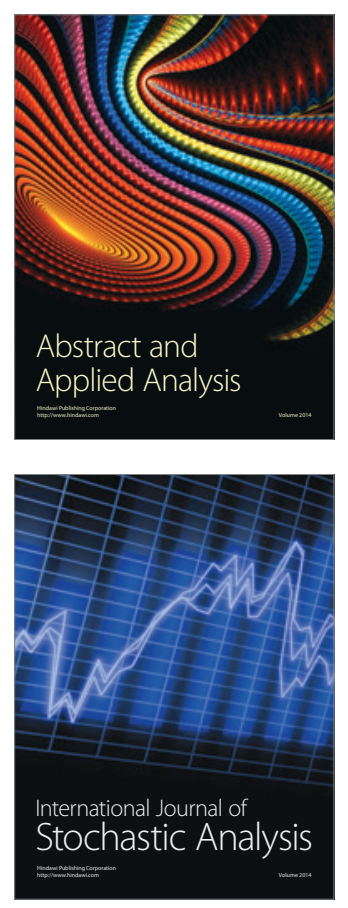

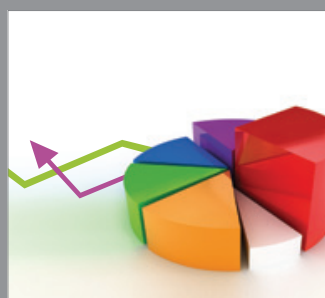

ournal of

Probability and Statistics

Promensencen
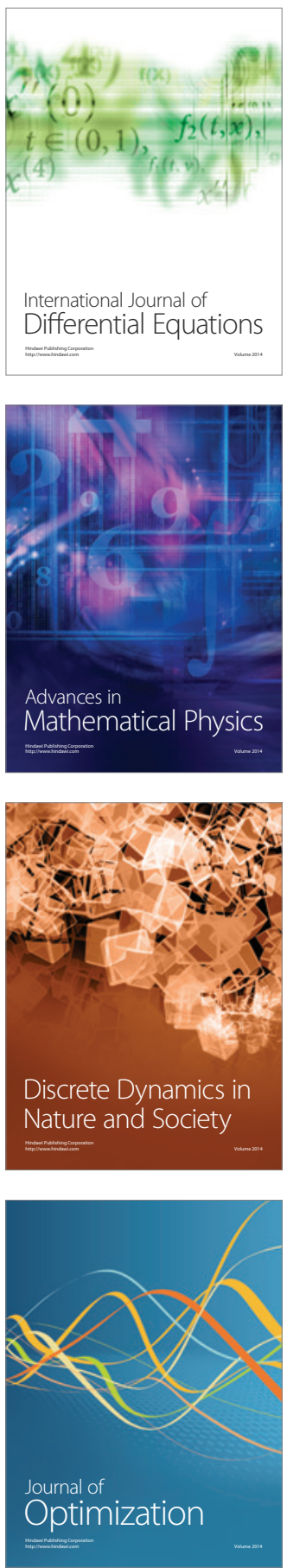